\title{
Investigation on structural, dielectric and ferroelectric properties of samarium-substituted $\mathrm{BiFeO}_{3}-\mathrm{PbTiO}_{3}$ composites
}

\author{
Truptimayee Sahu* and Banarji Behera \\ Material Research Laboratory, School of Physics, Sambalpur University \\ Jyoti Vihar Burla, Sambalpur 768019, Odisha, India \\ *trupti.m.sahu90@gmail.com
}

Received 5 October 2016; Revised 3 November 2016; Accepted 28 December 2016; Published 9 February 2017

\begin{abstract}
The polycrystalline samples of $0.8 \mathrm{BiSm}_{x} \mathrm{Fe}_{1-x} \mathrm{O}_{3}-0.2 \mathrm{PbTiO}_{3}(x=0.05,0.10,0.15$ and 0.20$)$ were prepared by using the conventional solid-state reaction technique and sintered at high temperature $\left(850^{\circ} \mathrm{C}\right)$. X-ray diffraction $(\mathrm{XRD})$ confirms the distorted rhombohedral crystal structure for all the composites at room temperature. The surface morphology was checked by field-emission scanning electron microscope (FESEM) technique and homogeneous mixing of the components was confirmed by energydispersive analysis of X-ray (EDAX). The detailed study of dielectric properties of the composites reveals an increasing nature of dielectric constant $\left(\varepsilon_{r}\right)$ and loss tangent $(\tan \delta)$ with the increase of temperature due to thermal activation. The Arrhenius plots of temperature dependence of $\mathrm{AC}$ conductivity yield the activation energy within the material at high-temperature range. The ferroelectric study shows that the remnant polarization decreases with the increase of Samarium $(\mathrm{Sm})$ concentration.
\end{abstract}

Keywords: X-ray diffraction; composite materials; dielectric materials; ferroelectricity.

\section{Introduction}

Material exhibiting the coexistence of two or more ferroic orders is known as multiferroic material. Bismuth ferrite, $\mathrm{BiFeO}_{3}(\mathrm{BFO})$, is one such material that exhibits multiferroic behavior at room temperature with ferroelectric Curie temperature $T_{C} \sim 850^{\circ} \mathrm{C}$ and antiferromagnetic Neel temperature $T_{N} \sim 350^{\circ} \mathrm{C} .^{1,2} \mathrm{X}$-ray diffraction (XRD) and neutron diffraction techniques have confirmed the distorted perovskite structure with rhombohedral crystal symmetry with the space group of $R 3 c$ for $\mathrm{BFO},{ }^{3-5}$ in which ferroelectricity is due to the stereochemical activity of Bi lone electron pair and the ferromagnetism is due to the partially filled $3 d$-orbitals of $\mathrm{Fe}^{3+}$ ion. ${ }^{6,7}$ But, due to high leakage current, impurity phases, oxygen vacancies and valence fluctuation of $\mathrm{Fe}$ ion $\left(\mathrm{Fe}^{3+}\right.$ and $\mathrm{Fe}^{2+}$ ) hindered this material for possible device application. In order to overcome these limitations, different researchers doped various rare earths on A- or B-site of perovskite material and also tried to make composites with other perovskite materials. $\mathrm{BiFeO}_{3}-\mathrm{PbTiO}_{3}$ (BFO-PTO) is one such perovskite composite observed at room temperature. ${ }^{8,9}$ This material also shows some interesting property. It shows morphotrophic phase boundary (MPB). The MPB in $(1-x) \mathrm{BFO}-(x) \mathrm{PTO}$ exists at $x=0.30$ with the simultaneous existence of rhombohedral $(R 3 \mathrm{c})$ and tetragonal $(\mathrm{P} 4 \mathrm{~mm})$ phases. However, the width of MPB is uncertain. The maximum width of MPB is at $x=0.20-0.31 .{ }^{10}$ The polycrystalline sample of BFO-PTO has achieved tremendous attention among researchers for its huge industrial applications due to the increase in multiferroic activity. ${ }^{11-14}$
Recently, some modification of these samples is being used for various applications such as actuators, transducers, sensors, multiple state memories, spintronics, etc. ${ }^{15,16}$ Therefore, in order to know the behavior of $0.8 \mathrm{BFO}-0.2 \mathrm{PTO}$, which is very close to MPB, we have reported the structural, dielectric and ferroelectric properties of $0.8 \mathrm{BiSm}_{x} \mathrm{Fe}_{1-x} \mathrm{O}_{3}-0.2 \mathrm{PbTiO}_{3}$ with $x=0.05,0.10,0.15$ and 0.20 , prepared by using the conventional solid-state reaction technique.

\section{Experimental Technique}

The polycrystalline samples of $0.8 \mathrm{BiSm}_{x} \mathrm{Fe}_{1-x} \mathrm{O}_{3}-0.2 \mathrm{PbTiO}_{3}$ with $x=0.05,0.10,0.15$ and 0.20 were prepared by conventional solid-state reaction technique. Stoichiometric amount of high-purity solid oxides: $\mathrm{Bi}_{2} \mathrm{O}_{3}(99.0 \%), \mathrm{Sm}_{2} \mathrm{O}_{3}$ (99.9\%), $\mathrm{PbO}$ (98.0\%; Central Drug House Pvt. Ltd., India), $\mathrm{Fe}_{2} \mathrm{O}_{3}$ (95-98\%; Loba Chemicals Pvt. Ltd., India) and $\mathrm{TiO}_{2}$ ( $\geq 99.0 \%$; Merck Specialities Pvt. Ltd., India) were mixed thoroughly using an agate mortar for $2 \mathrm{~h}$ and then in alcohol medium for another $2 \mathrm{~h}$. The mixed samples were calcined in a high-purity alumina crucible at $825^{\circ} \mathrm{C}$ for $5 \mathrm{~h}$ in an air atmosphere. The phase formation of the composites were checked by XRD (RIGAKU Ultima IV) by using $\mathrm{Cu}-\mathrm{K}_{\alpha}$ radiation $(\lambda=1.5405 \AA)$ in a wide range of Bragg's angle $2 \theta$ $\left(20^{\circ} \leq 2 \theta \leq 80^{\circ}\right)$ at a scanning rate of $3 \% \mathrm{~min}$. The processes of grinding and calcination were repeated for several times until the phase formation of the compound was confirmed. Then polyvinyl alcohol (PVA) was mixed with the calcined powder, which acts as a binder and burnt out during sintering.

This is an Open Access article published by World Scientific Publishing Company. It is distributed under the terms of the Creative Commons Attribution 4.0 (CC-BY) License. Further distribution of this work is permitted, provided the original work is properly cited. 
By using an hydraulic press, the calcined powders are pressed into cylindrical pellets of $12 \mathrm{~mm}$ diameter and $1-2 \mathrm{~mm}$ thickness at a pressure of about $3.5 \times 10^{6} \mathrm{~N} / \mathrm{m}^{2}$. Then the pellets were sintered at $850^{\circ} \mathrm{C}$ for $5 \mathrm{~h}$. In order to check the surface morphology of the composites the sintered pellets were studied by using field-emission scanning electron microscope (FESEM) technique (FEI ESEM QUANTA 200 3D). The sintered pellets were polished by fine emery paper to make both the surfaces flat and parallel. The pellets were electroded with air-drying conducting silver paste to study the electrical properties. Then the pellets were dried at $150^{\circ} \mathrm{C}$ for $2 \mathrm{~h}$ to remove moisture, if present, and cooled to room temperature before taking any electrical measurement. The dielectric properties of the composites were measured by using an LCR meter (HIOKI, Model-3532) in a wide range of frequencies $\left(10^{2}-10^{6} \mathrm{~Hz}\right)$ and temperatures $\left(25-450^{\circ} \mathrm{C}\right)$. The ferroelectric properties were investigated by means of a computer-based automatic PE-loop tracer (Marine India, India).

\section{Results and Discussion}

\subsection{X-ray diffraction analysis}

The crystal structures associated with $0.8 \mathrm{BiSm}{ }_{x} \mathrm{Fe}_{1-x} \mathrm{O}_{3}-$ $0.2 \mathrm{PbTiO}_{3}$ samples with $x=0.05,0.10,0.15$ and 0.20 were estimated with the help of XRD pattern obtained from the powder sample. Figure 1 shows the XRD patterns of the composites at room temperature. These patterns do not show the individual peaks of $\mathrm{BiFeO}_{3}$ (BFO) and $\mathrm{PbTiO}_{3}$ (PTO), which suggests the presence of intermixing of different phases during mixing and heating. In XRD patterns, some additional peaks have been identified and indexed in Fig. 1. The secondary phases of $\mathrm{Bi}_{2} \mathrm{Fe}_{4} \mathrm{O}_{9}, \mathrm{Bi}_{25} \mathrm{FeO}_{40}$ and $\mathrm{FeSmO}_{3}$ were confirmed by JCPDF file nos. 741-098, 460-416 and 391-490, respectively. Further, these secondary phases do not make any effect on the crystal symmetry, and crystal structures of the studied samples were consistent with distorted rhombohedral crystal symmetry. Because, according to

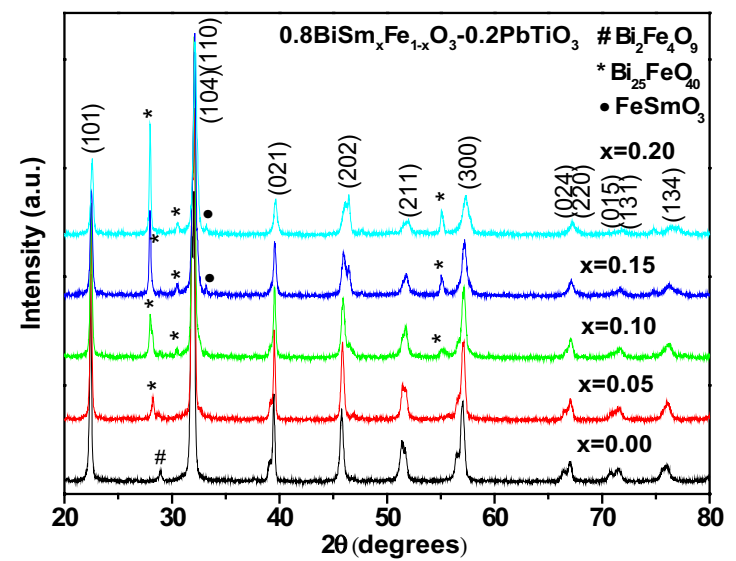

Fig. 1. XRD patterns of $0.8 \mathrm{BiSm}_{x} \mathrm{Fe}_{1-x} \mathrm{O}_{3}-0.2 \mathrm{PbTiO}_{3}(x=0.05$, $0.10,0.15$ and 0.20 ). first-principles study, ${ }^{17}$ it has been reported that the structural stability in perovskite oxide is due to stoichiometric balance between the long-range $\mathrm{A}-\mathrm{O}$ bond and short-range $\mathrm{B}-\mathrm{O}$ bond forces, where the long-range forces are mainly responsible for causing large strain in the lattice and that causes the asymmetric structural arrangement in the lattice. But the shortrange $\mathrm{B}-\mathrm{O}$ bond tries to stabilize the perovskite structure to cubic phase. ${ }^{18}$ Here we may say that, in BF-PT composite, both $\mathrm{Bi}-\mathrm{O}$ and $\mathrm{Pb}-\mathrm{O}$ bonds make possible hybridization and this results in distorted symmetry in its structure. This may be due to the larger atomic radios of $\mathrm{Sm}^{3+}(0.124 \mathrm{~nm})^{19}$ than that of $\mathrm{Fe}^{3+}(0.0785 \mathrm{~nm})$ that affects the short-range $\mathrm{B}-\mathrm{O}$ bond. One literature survey shows that the composites $(1-x)$ BF- $x$ PT show rhombohedral phase with $x \leq 0.27$ and for $x=0.30$ show MPB having both rhombohedral and tetragonal phases. ${ }^{20}$ The detailed crystal structure was studied by using a standard computer program package $P O W D .^{21}$ The refinement was performed by using the rhombohedral lattice with lattice constant $a=b=5.58 \AA$ and $c=13.8 \AA .^{20}$ The lattice parameters were obtained by refinement through leastsquares subroutine method. This process was continued until a good agreement between the observed and calculated $d$-spacing, i.e., $\sum \Delta d=d_{\text {obs }}-d_{\text {cal }}=$ minimum. The different lattice parameters observed are listed in Table 1. This data shows that the $c / a$ ratio decreases with increase of Sm concentration which indicates the more symmetrical structure above $x=0.20$. The $c / a$ ratios obtained for the samples are in good agreement with the reported one ${ }^{22}$ for 0.8 BFO- 0.2 PTO prepared by using chemical method. The crystallite size of the composites was measured by using Scherrer's equation $P=K \lambda /\left(\beta_{1 / 2} \cos \theta_{h k l}\right)$, where the constant $K=0.89$, $\lambda=1.5405 \AA$ and $\beta_{1 / 2}$ is the value of full width at half intensity of the peaks. The average values of crystallite size have been obtained within the range of $25-30 \mathrm{~nm}$ for all the composites.

\subsection{Scanning electron microscopy}

The surface morphology of the sintered samples was investigated by FESEM technique and the obtained images are compared in Figs. 2(a)-2(d). According to the images, cubical shape grains having a grain size within $0.5-1.5 \mu \mathrm{m}$ range were observed. These micrographs also demonstrate homogeneous grains with high density. This confirms the polycrystalline

Table 1. Different lattice parameters (in $\AA$ ) of $0.8 \mathrm{BiSm}_{x} \mathrm{Fe}_{1-x} \mathrm{O}_{3}-0.2 \mathrm{PbTiO}_{3}$ $(x=0.05,0.10,0.15$ and 0.20$)$.

\begin{tabular}{lrrrr}
\hline Lattice parameters & \multicolumn{4}{c}{ Sm concentration in $0.8 \mathrm{BiSm}_{x} \mathrm{Fe}_{1-x} \mathrm{O}_{3}-0.2 \mathrm{PbTiO}_{3}$} \\
\cline { 2 - 5 } & $x=0.05$ & $x=0.10$ & $x=0.15$ & $x=0.20$ \\
\hline$a$ & 5.5713 & 5.5725 & 5.5725 & 5.5670 \\
$c$ & 13.8222 & 13.8200 & 13.8178 & 13.7188 \\
$c / a$ & 2.4810 & 2.4800 & 2.4796 & 2.4643 \\
\hline
\end{tabular}




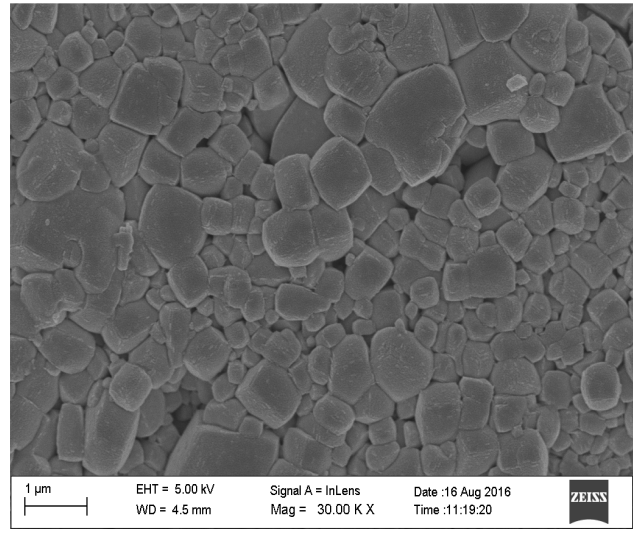

(a)

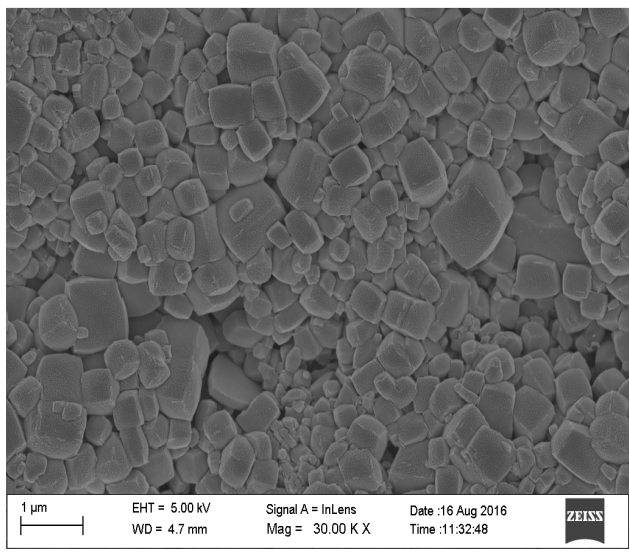

(c)

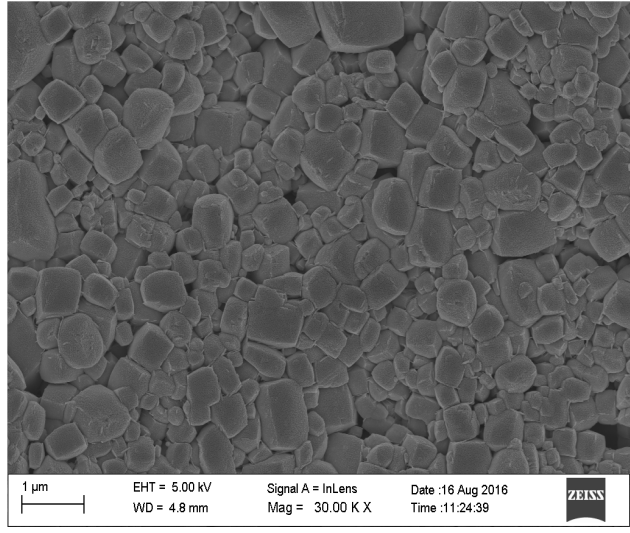

(b)

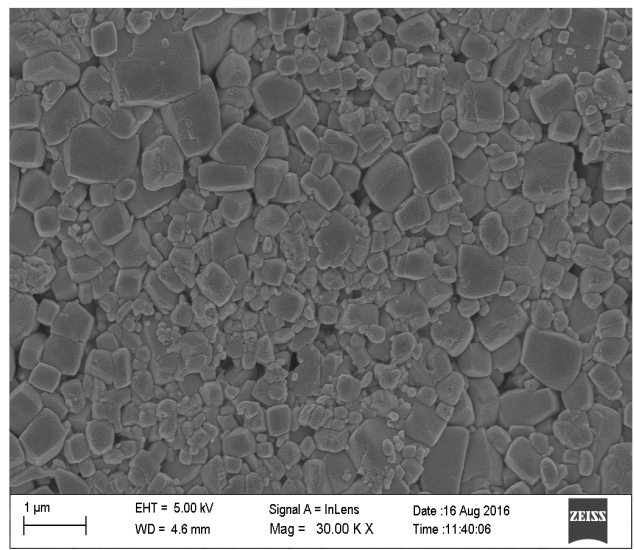

(d)

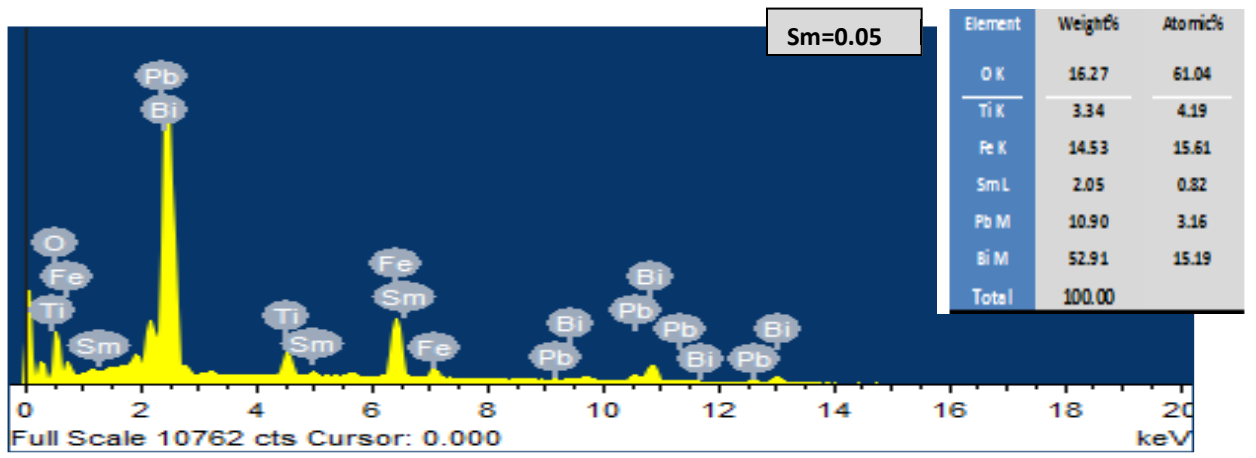

(e)

Fig. 2. (a)-(d) FESEM images of $0.8 \mathrm{BiSm}_{x} \mathrm{Fe}_{1-x} \mathrm{O}_{3}-0.2 \mathrm{PbTiO}_{3}(x=0.05,0.10,0.15$ and 0.20 ), respectively, and Panel (e) shows the EDAX pattern for $0.8 \mathrm{BiSm}_{0.05} \mathrm{Fe}_{0.95} \mathrm{O}_{3}-0.2 \mathrm{PbTiO}_{3}$.

nature of the composites. The energy dispersive analysis of X-ray (EDAX) result of $0.8 \mathrm{BiSm}_{0.05} \mathrm{Fe}_{0.95} \mathrm{O}_{3}-0.2 \mathrm{PbTiO}_{3}$ (Fig. 2(e)) shows the homogeneous mixing and phase formation within the material.

\subsection{Ferroelectric properties}

The room-temperature ferroelectric hysteresis loops of 0.8 $\mathrm{BiSm}_{x} \mathrm{Fe}_{1-x} \mathrm{O}_{3}-0.2 \mathrm{PbTiO}_{3}(x=0.05,0.10,0.15$ and 0.20$)$ obtained by applying a maximum field of $9 \mathrm{kV} / \mathrm{cm}$ and an applied frequency of $50 \mathrm{~Hz}$ are shown in Figs. 3(a) and 3(b). The remnant polarization $\left(2 P_{r}\right)$ of the above composites decreases with the increase of $\mathrm{Sm}$ concentration, i.e., 0.232, $0.163,0.142$ and $0.088 \mu \mathrm{C} / \mathrm{cm}^{2}$ for $x=0.05,0.10,0.15$ and 0.20 , respectively. This may be due to large difference in ionic radii of $\mathrm{Sm}^{3+}$ and $\mathrm{Fe}^{3+}$, as it is well known that polarization in ferroelectrics is very sensitive to structural distortions or changes in chemical bonds within the material. 


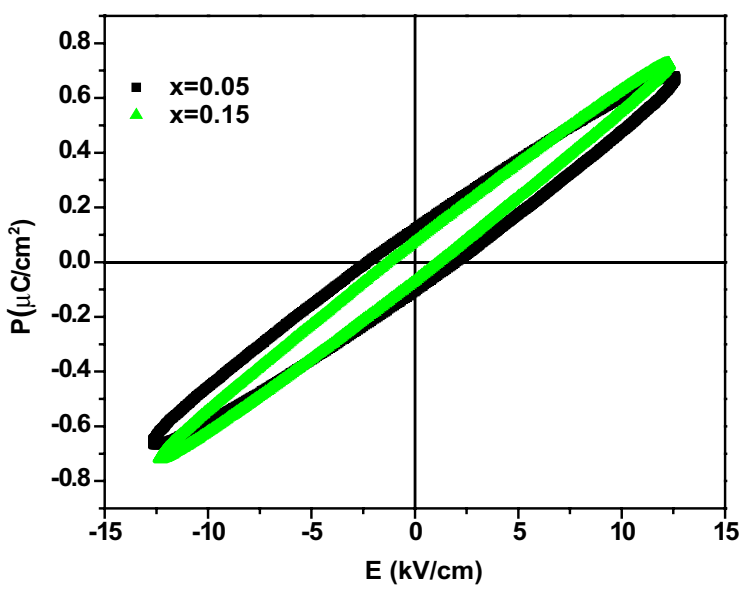

(a)

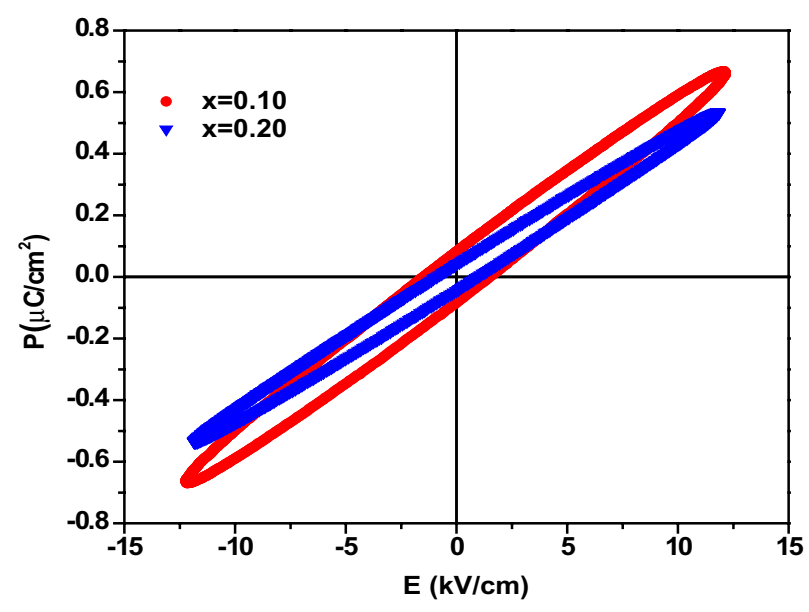

(b)

Fig. 3(a-b). Ferroelectric hysteresis loops for unpoled sample of $0.8 \mathrm{Bi}_{x} \mathrm{Fe}_{1-x} \mathrm{O}_{3}-0.2 \mathrm{PbTiO}_{3}(x=0.05,0.10,0.15$ and 0.20$)$ at room temperature.

The above values are obtained for unpoled samples and can withstand a field more than $20 \mathrm{kV} / \mathrm{cm}$.

\subsection{Dielectric properties study}

The frequency-dependent dielectric constant $\left(\varepsilon_{r}\right)$ variations at $275^{\circ} \mathrm{C}$ and $25^{\circ} \mathrm{C}$ are shown in Fig. 4 and its inset, respectively. The dielectric constant decreases smoothly with frequency and shows large dispersion at high-frequency region, which is the usual behavior of dielectric materials. ${ }^{23}$ The high value of dielectric constant at low-frequency region is due to the simultaneous presence of all types of polarization (i.e., electronic, ionic, atomic and space charge polarizations) which align themselves in one direction with the applied field. But at high-frequency region dipoles cannot synchronize with frequency of the applied field; so the contribution of polarization reduces and appears to merge into a single line with very low value of dielectric constant at high-frequency

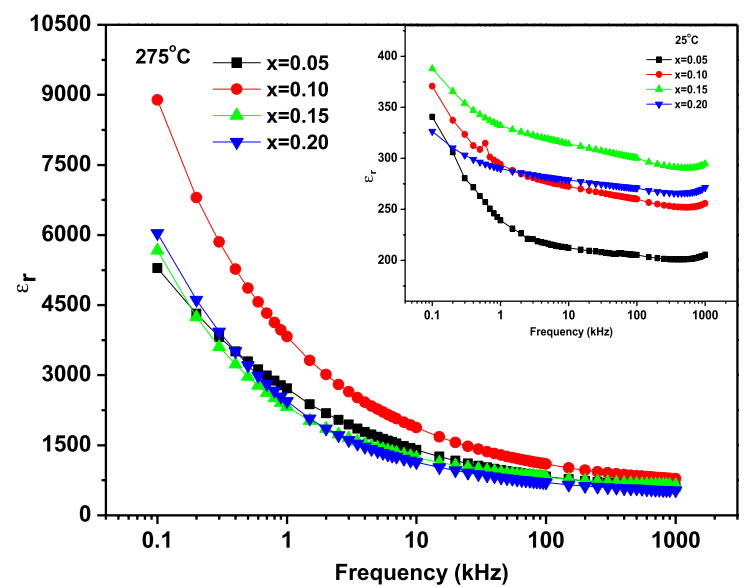

Fig. 4. Variations of $\varepsilon_{r}$ with frequency at $275^{\circ} \mathrm{C}$ and $25^{\circ} \mathrm{C}$ (inset) of $0.8 \mathrm{BiSm}_{x} \mathrm{Fe}_{1-x} \mathrm{O}_{3}-0.2 \mathrm{PbTiO}_{3}(x=0.05,0.10,0.15$ and 0.20$)$. region. ${ }^{24}$ The grain boundary is more active at lowfrequencies and grains are more active at high-frequencies. ${ }^{25}$ Thus, at low-frequency region, grains are more active and possess high resistance. So, more energy is required for polarization of charge carriers and hence, energy loss is also high. In contradiction, at high-frequency region, grain boundaries are less active so less energy is required for polarization and hence energy loss is also less. This typical behavior of loss tangent at $275^{\circ} \mathrm{C}$ with frequency is shown in Fig. 5. From this graph we can say that the materials have perfectly conducting grains separated by well-defined grain boundaries at low frequencies. The temperature dependent of dielectric constant $\left(\varepsilon_{r}\right)$ and loss tangent $(\tan \delta)$ at $100 \mathrm{kHz}$ are shown in Fig. 6 and Fig. 6 (inset) respectively. Both $\varepsilon_{r}$ and $\tan \delta$ increase with temperature due to the increase of conduction electrons due to thermal activation. ${ }^{26}$ No dielectric anomaly has been observed for the studied composites for selected range of temperatures $\left(25-450^{\circ} \mathrm{C}\right)$.

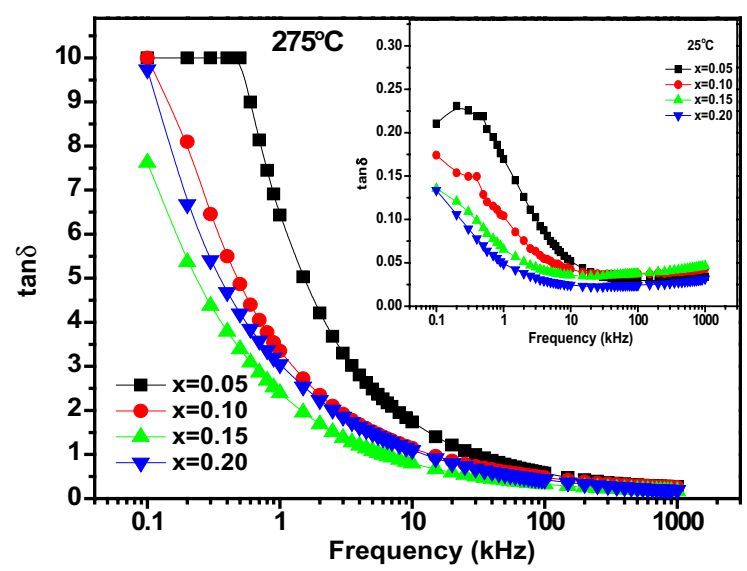

Fig. 5. Frequency variations of $\tan \delta$ at $275^{\circ} \mathrm{C}$ and $25^{\circ} \mathrm{C}$ (inset) of $0.8 \mathrm{BiSm}_{x} \mathrm{Fe}_{1-x} \mathrm{O}_{3}-0.2 \mathrm{PbTiO}_{3}(x=0.05,0.10,0.15$ and 0.20$)$. 


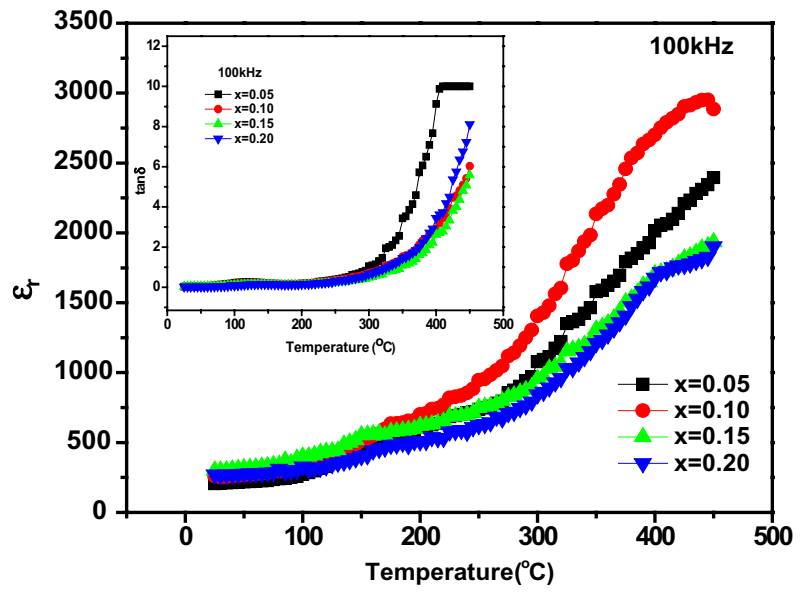

Fig. 6. Variations of $\varepsilon_{r}$ and $\tan \delta$ (inset) with respect to temperature at $100 \mathrm{kHz}$ of $0.8 \mathrm{BiSm}_{x} \mathrm{Fe}_{1-x} \mathrm{O}_{3}-0.2 \mathrm{PbTiO}_{3}(x=0.05,0.10,0.15$ and 0.20$)$.

\subsection{Conductivity study}

The AC conductivity obtained from dielectric study was plotted as a function of inverse temperature at $50 \mathrm{kHz}$ as shown in Fig. 7. The observed value of AC conductivity was calculated by using the empirical relation $\sigma_{\mathrm{ac}}=\omega \varepsilon_{0} \varepsilon_{r} \tan \delta$, where $\omega$ is the angular frequency, $\varepsilon_{0}$ is the permitivity in free space and $\tan \delta$ is the loss tangent. One can also define the conductivity as the amount of energy required for the conduction of charge carriers from valence band to conduction band, i.e., the activation energy indicates about the conduction process occuring within the material. The AC conductivity follows Arrhenius equation $\sigma_{\mathrm{ac}}=\sigma_{0} \exp \left(-E_{a} / K_{B} T\right)$, where $\sigma_{0}$ is the pre-exponential factor, $K_{B}$ is the Boltzmann's constant and $E_{a}$ is the activation energy. In Fig. 7, at higher temperature range, the activation energies were found to be $0.7085,0.7346,0.7497$ and $0.7708 \mathrm{eV}$ for $x=0.05,0.10$, 0.15 and 0.20 , respectively.

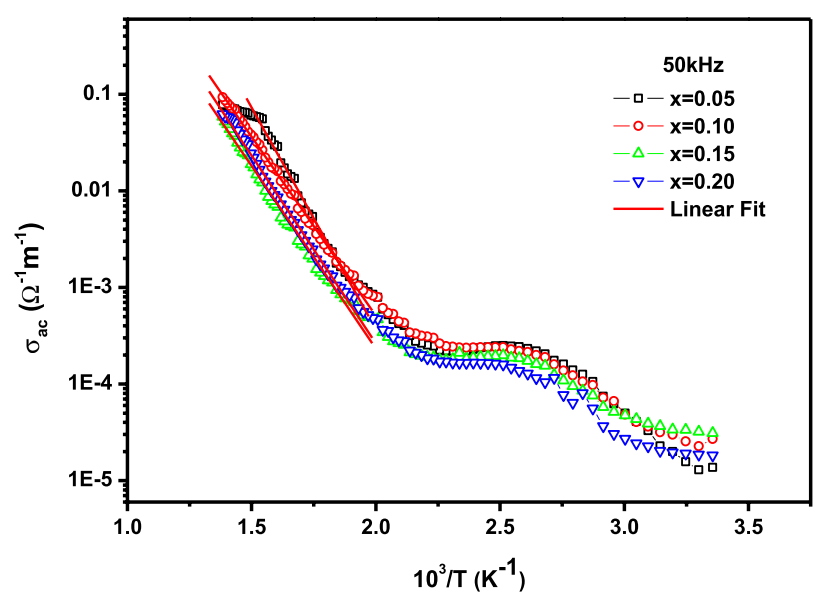

Fig. 7. Variations of $\mathrm{AC}$ conductivity $\left(\sigma_{\mathrm{ac}}\right)$ with inverse temperature $\left(10^{3} / T\right)$ for $0.8 \mathrm{Bi}_{x} \mathrm{Fe}_{1-x} \mathrm{O}_{3}-0.2 \mathrm{PbTiO}_{3}(x=0.05,0.10,0.15$ and 0.20 ) at a fixed frequency of $50 \mathrm{kHz}$.

\section{Conclusions}

The polycrystalline samples of $0.8 \mathrm{Bi}_{x} \mathrm{Fe}_{1-x} \mathrm{O}_{3}-0.2 \mathrm{PbTiO}_{3}$ with $(x=0.05,0.10,0.15$ and 0.20$)$ were prepared by using the conventional solid-state reaction method. All the studied samples were consistent with the same distorted rhombohedral (R3c) crystal symmetry. A decreasing trend of lattice parameters $(c / a$ ratio) has been observed with increase in $x$ which indicates the occurrence of a more stable structure above $x=0.20$. The remnant polarization decreases with the increase of $\mathrm{Sm}$ concentration due to smaller ionic radius of $\mathrm{Sm}$ than that of Fe. No dielectric anomaly has been observed within the selected range of temperatures. But, an enhancement in resistivity has been observed with increase in $\mathrm{Sm}$ concentration as the values of $\varepsilon_{r}$ and $\tan \delta$ decrease. The AC conductivity obtained from the dielectric data exhibits Arrhenius-type electrical conductivity and the value of $E_{a}$ increases with increase in $x$.

\section{Acknowledgments}

One of the authors (Truptimayee Sahu) acknowledges the financial support through DST-INSPIRE Fellowship to carry out the research work. The author also acknowledges the financial support through DRS-1 of UGC, New Delhi, India, under the SAP and FIST Programmes of DST, New Delhi, India, for the development of research work at School of Physics, Sambalpur University, Odisha. One of the authors (B. Behera) acknowledges the SERB, New Delhi, India, under DST Fast Track Scheme for Young Scientist (Project No. SR/FTP/PS-036/2011).

\section{References}

${ }^{1}$ F. Kubel and H. Schmid, Structure of a ferroelectric and ferroelastic monodomain crystal of the perovskite $\mathrm{BiFeO}_{3}$, Acta Crystallogr. B 46, 698 (1990).

${ }^{2}$ J. D. Bucci, B. K. Robertson and W. D. James, The precision determination of the lattice parameters and the coefficients of thermal expansion of $\mathrm{BiFeO}_{3}$, J. Appl. Cryst. 5, 187 (1972).

${ }^{3}$ C. Michel, J. M. Moreau, G. D. Achenbach, R. Gerson and W. J. James, The atomic structure of $\mathrm{BiFeO}_{3}$, Solid State Commun. 9, 701 (1969).

${ }^{4}$ P. Fischer, M. Polomska, I. Sosnowska and M. Szymanski, Temperature dependence of the crystal and magnetic structures of $\mathrm{BiFeO}_{3}$, J. Phys. C 13, 1931 (1980).

${ }^{5}$ J. R. Teague, R. Gerson and W. J. James, Dielectric hysteresis in single crystal $\mathrm{BiFeO}_{3}$, Solid State Commun. 8, 1073 (1970).

${ }^{6}$ P. K. Siwach, H. K. Singh, J. Singh and O. N. Srivastava, Anomalous ferromagnetism in spray pyrolysis deposited multiferroic $\mathrm{BiFeO}_{3}$ films, Appl. Phys. Lett. 91, 2503 (2007).

${ }^{7}$ V. A. Khomchenko, M. Kopcewicz, A. M. L. Lopes, Y. G. Pogorelov, J. P. Araujo, J. M. Vieira and A. L. Kholkin, Intrinsic nature of the magnetization enhancement in heterovalently doped $\mathrm{Bi}_{1-x} \mathrm{~A}_{x} \mathrm{FeO}_{3}(A=\mathrm{Ca}, \mathrm{Sr}, \mathrm{Pb}, \mathrm{Ba})$ multiferroics, J. Phys. D, Appl. Phys. 41, 102003 (2008). 
${ }^{8} \mathrm{~S}$. Bhattacharjee and D. Pandey, Stability of the various crystallographic phases of the multiferroic $(1-x) \mathrm{BiFeO}_{3}-x \mathrm{PbTiO}_{3}$ system as a function of composition and temperature, J. Appl. Phys. 107, 124112 (2010).

${ }^{9}$ R. Ranjan and K. A. Raju, Unconventional mechanism of stabilization of a tetragonal phase in the perovskite ferroelectric $\left(\mathrm{PbTiO}_{3}\right)_{1-x}\left(\mathrm{BiFeO}_{3}\right)_{x}$, Phys. Rev. B 82, 054119 (2010).

${ }^{10}$ W. M. Zhu, H. Y. Guo and Z. G. Ye, Structural and magnetic characterization of multiferroic $\left(\mathrm{BiFeO}_{3}\right)_{1-x}-\left(\mathrm{PbTiO}_{3}\right)_{x}$ solid solutions, Phys. Rev. B 78, 014401 (2008).

${ }^{11} \mathrm{~J}$. Cheng and Z. Meng, High-field and high- $T_{c}$ piezoelectric ceramics based on $\mathrm{Bi}(\mathrm{Ga}, \mathrm{Fe}) \mathrm{O}_{3}-\mathrm{PbTiO}_{3} \mathrm{Bi}(\mathrm{Ga}, \mathrm{Fe}) \mathrm{O}_{3}-\mathrm{PbTiO}_{3}$ crystalline solutions, J. Appl. Phys. 98, 084102 (2005).

${ }^{12}$ M. A. Khan, T. P. Comyn and A. J. Bell, Ferroelectric $\mathrm{BiFeO}_{3}-$ $\mathrm{PbTiO}_{3}$ thin films on $\mathrm{Pt} / \mathrm{Si}$ substrates, IEEE Trans. Ultrason. Ferroelectr. Freq. Control 54, 2583 (2007).

${ }^{13}$ J. Bennett, T. R. Shrout, S. J. Zhang, P. Mandal, A. J. Bell, T. J. Stevenson and T. P. Comyn, Temperature dependence of the intrinsic and extrinsic contributions in $\mathrm{BiFeO}_{3}-\left(\mathrm{K}_{0.5} \mathrm{Bi}_{0.5}\right) \mathrm{TiO}_{3}-$ $\mathrm{PbTiO}_{3}$ piezoelectric ceramics, J. Appl. Phys. 116, 094102 (2014).

${ }^{14}$ T. P. Comyn, T. Stevenson, M. A. Jawad, W. G. Marshall, R. I. Smith, J. H. Albillos, R. Cywinski and A. J. Bell, Pressure induced para-antiferromagnetic switching in $\mathrm{BiFeO}_{3}-\mathrm{PbTiO}_{3}$ as determined using in-situ neutron diffraction, J. Appl. Phys. 113, 183910 (2013).

${ }^{15}$ M. Fiebig, T. Lottermoser, D. Frohlich, A. V. Goitsev and R. V. Pisarev, Observation of coupled magnetic and electric domains, Nature 419, 818 (2002).

${ }^{16}$ J. Wang, J. B. Neaton, H. Jheng, V. Nagarjan, S. B. Ogale, B. Liu, D. Viehland, V. Vaithyanathan, D. G. Schlom, U. V. Waghmare,
N. A. Spaldin, K. M. Rabe, M. Wuttig and R. Ramesh, Epitaxial $\mathrm{BiFeO}_{3}$ multiferroic thin film heterostructures, Science 299, 1719 (2003).

${ }^{17}$ N. A. Hill and A. Filippetti, Why are there any magnetic ferroelectrics? J. Magn. Magn. Mater. 242, 976 (2002).

${ }^{18} \mathrm{R}$. Cohen, Origin of ferroelectricity in perovskite oxides, Nature 358, 136 (1992).

${ }^{19}$ A. Mukherjee, S. Basu, P. K. Manna, S. M. Yusuf and M. Pal, Giant magnetodielectric and enhanced multiferroic properties of Sm doped bismuth ferrite nanoparticles, J. Mater. Chem. C 2, 5885 (2014).

${ }^{20} \mathrm{~S}$. Bhattacharjee, S. Tripathi and D. Pandey, Morphotropic phase boundary in $(1-x) \mathrm{BiFeO}_{3}-x \mathrm{PbTiO}_{3}$ : Phase coexistence region and unusually large tetragonality, Appl. Phys. Lett. 91, 042903 (2007).

${ }^{21}$ E. Wu, POWDMULT: An Interactive Powder Diffraction Data Interpretation and Indexing Program, Ver. 2.1, School of Physical Science, Flinders University, Australia (1989).

${ }^{22}$ A. Siddaramanna et al., Stabilization of metastable tetragonal phase in a rhombohedral magnetoelectric multiferroic $\mathrm{BiFeO}_{3}-$ $\mathrm{PbTiO}_{3}$, J. Phys. D, Appl. Phys. 47, 045004 (2014).

${ }^{23} \mathrm{E}$. İzci and A. İzci, Dielectric behavior of the catalyst zeolite NaY, J. Chem. 31, 523 (2007).

${ }^{24}$ C. J. F. Bottchar, Theory of Electric Polarization (Elsevier, Amsterdam, 1952).

${ }^{25} \mathrm{C}$. G. Koops, On the dispersion of resistivity and dielectric constant of some semiconductors at audio frequencies, Phys. Rev. 83, 121 (1951).

${ }^{26}$ P. Q. Mantus, Dielectric response of materials: Extension to the Debye model, J. Eur. Ceram. Soc. 19, 2079 (1999). 